\title{
Engineering optimization of decompressive craniectomy based on finite element simulations
}

\author{
MÁTÉ HAZAY ${ }^{1 *}$, EMESE NAGY ${ }^{1}$, PÉTER TÓTH ${ }^{2}$, ANDRÁS BÜKI $^{2}$, IMRE BOJTÁR ${ }^{1}$ \\ ${ }^{1}$ Department of Structural Mechanics, Budapest University of Technology and Economics, Budapest, Hungary. \\ ${ }^{2}$ Department of Neurosurgery, University of Pecs, Pecs, Hungary.
}

\begin{abstract}
Purpose: The optimal execution of decompressive craniectomy in terms of the size and location of the skull opening is not straightforward. Our main goals are twofold: (1) constructing a design optimization method which can be applied to determine optimal skull opening for individual patient-specific cases and (2) performing a large-scale parametric optimization study to give some guidance in general about the optimal skull opening in case of oedematous brain tissue. Methods: A large number of virtual experiments performed by finite element simulations were applied to determine tendencies of tissue behaviour during surgery. The multiobjective optimization is performed by Goal Programming and Physical Programming methods. Results: Our results show that the postoperative pressure has an approximately linear dependence on the preoperative pressure and the skull opening area, while the damaged brain volume could have a more complex nonlinear dependence on the input data. Based on the averaged results of the parametric optimization study, the optimal skull opening has been determined in the function of the preoperative pressure and the relative importance of the pressure reduction. These results show that the optimal size of the unilateral skull opening is usually between $130-180 \mathrm{~cm}^{2}$ and these openings are more beneficial than the currently analysed bifrontal openings. Conclusions: The optimal skull opening is patient-specific and depends on several input data. The presented methodology can be applied to optimize surgery based on these input parameters for different injury types. Based on the results of large-scale parametric study generally applicable approximate results have been provided.
\end{abstract}

Key words: decompressive craniectomy, computer-assisted neurosurgery, engineering optimization, finite element head models

\section{Introduction}

Traumatic brain injuries (TBIs) are a major health problem worldwide due to the large number and the severity of these cases. In the United States, 1.7 million people suffer a TBI every year [5], and the $2 \%$ of the population (5.3 million citizens) are living with disability as a result of TBI [23]. The annual cost of these injuries is more than 60 billion dollars [16], implying that the treatment and rehabilitation of the injured people cause a considerate economic burden to society.

After TBI, medical and surgical therapies are performed to avoid or minimize secondary brain injury. Increased intracranial pressure (ICP), which is typi- cally caused by cerebral oedema, is the most frequent cause of further neurological deterioration and death after a severe head injury [21]. Oedema has significant epidemiological importance as together with its complications they account for approximately $50 \%$ of TBI related deaths [17]. Medical and surgical therapies fit into a tiered treatment procedure. In conjunction with monitoring of ICP, first-tier methods, such as sedation and adjustment of body temperature or carbon dioxide levels in the blood may mitigate ICP indirectly [21] precluding the use of any surgical intervention. However, if the elevated ICP cannot be controlled by first-, and second-tier methods, then third tier interventions, such as barbiturate coma or decompressive craniectomy (DC), can be applied. In case of DC, a relatively large part of the skull is re-

\footnotetext{
* Corresponding author: Máté Hazay, Department of Structural Mechanics, Budapest University of Technology and Economics, Hungary, Müegyetem rkp. 3., 1111 Budapest, Hungary. E-mail: hazay.mate@epito.bme.hu

Received: June 29th, 2020

Accepted for publication: September 28th, 2020
} 
moved, and the underlying dura mater is opened to allow a bulging deformation of the brain tissue.

DC is proved to be an effective tool for reducing ICP, however, its overall effectiveness is not so straightforward because the brain tissue may suffer extreme deformations during surgery. Although the tissue damage may not be observable during surgery, it is thought that the excessive stretching and shear torsion of the axonal fibres can contribute to an unfavourable outcome [3]. This has been analysed in large-scale clinical trials, and it was found that surgical treatment sometimes could be associated with better [14] and worse [3] outcome than the standard medical care. This outcome mainly depends on the preoperative pressure and the stage of the tiered treatment when the DC was executed. Recent evidence substantiated from the RESCUEicp Trial [14] provided additional scientific support for the beneficial role of DC in ICP control also pointing to its contribution to better long-term outcome. Accordingly, DC is increasingly performed in many neurotrauma centres internationally [21], and probably this remains the case in the future as well. However, because of the complexity of the problem and the fact that not just medical but biomechanical factors should also be considered during the decision-making, the optimal execution of DC is not straightforward. Certain aspects in terms of the size and location of the skull opening are controversial [6], and currently, the applied skull opening is mostly based on personal experience. Some failures in clinical trials might as well be associated with lack of appreciation of different tissue properties, and shearing forces during brain tissue expansion upon craniectomies performed at various regions. These findings and the above considerations imply that there is a need for better understanding of tissue behaviour during surgery, and the optimization of DC could be identified as an important research goal harbouring significant medical and biomechanical aspects. In this paper, the engineering optimization of DC is performed from a biomechanical point of view, with the hope that the engineering observations of the tissue behaviour and the obtained results regarding the optimal skull opening could support the decisionmaking during the design of DC.

Despite the enormous complexity of the brain, many aspects of its physical response and behaviour can be described purely by mechanical terms, such as displacements, strains and stresses. Although several engineering research works have been performed focusing on different brain lesions like hydrocephalus and tumor resection [19], relatively few biomechanical research has been carried out to analyse oedema and DC. From the modelling point of view, the current field of investigation significantly differs from previous studies [19] due to the large bulging displacements and strains which may occur during DC due to the excessive ICP. The first 3D finite element (FE) head model to analyse DC was developed by Gao and Ang [10]. They performed a small number of virtual experiments with different skull openings. It was determined that the maximum stress regions can be found around the edges of the craniectomy. Furthermore, based on the observed ICPs and displacements, they concluded that an optimal skull opening should exist. Others [24] used nonlinear medical image registration (MIR) to determine the brain deformations during the preoperative and postoperative state founding significant strains, indicating that the loss of neuronal function is possible due to the operation. Later, patient-specific FE models were developed [15] to reconstruct real-life DC cases and it was found that FE models can reliably predict the motions of the brain surface during surgery. Afterwards, the results of FE models with simplistic geometry were compared to the observations obtained by an experimental model using gelatin hydrogel [6]. Moreover, their sensitivity analysis regarding the applied material models showed that FE models with elastic material behaviour adequately capture the peak strains during DC. Based on these findings, simplistic and realistic FE models were used with hyperelastic material model to analyse the effect of the chamfer at the skull edge [7], and it was found that its details have a relatively small effect on the strain field and the damaged brain volume.

Despite the effort of previous research works, currently, our knowledge regarding the optimal execution of DC is very limited. Previous FE models were not clinically validated [7], [10] or were validated only by comparison of displacements [15], while the agreement of ICPs was not considered. Comprehensive series of virtual experiments have not been performed yet for different realistic preoperative ICP values due to numerical difficulties, which became the limitation of previous research [7], [11]. Currently, only the existence of the optimization problem has been stated, but the exact formulation of the problem in terms of objective functions, goals and solution algorithms have not been proposed. Similarly, recommendations for the optimal skull opening based on the consideration of the biomechanical behaviour of the brain tissue have not been presented yet.

To improve our current understanding regarding the tissue behaviour during surgery, we aim to perform a large number of simulations with different skull 
openings on FE models which have realistic, patientspecific geometry. The applied modelling strategy, including the model development and the validation based on the reconstruction of real-life cases, have been detailed elsewhere [11], [12]. In this paper, the emphasis is taken on a large number of new FE results and the exact mathematical formulation of the optimization problem. In general, the optimal skull opening is patientspecific and depends on several input data. The proposed optimization method can be applied to optimize surgery in an individual, patient-specific manner based on these input parameters for different injury types. However, to provide generally applicable approximate results, the current optimization strategy is nested into a large-scale parametric study. Hence, the optimization is performed for multiple realizations of the input parameters and the average of these individual results are used as an estimate for the optimal skull opening.

\section{Materials and methods}

The currently applied method can be considered a modular system (Fig. 1). It starts with Module A, which includes the FE model development and its validation by the reconstruction of a real-life DC case [12]. Once validated FE models are available, a large number of virtual experiments are performed to analyse several DC scenarios (Module B in Fig. 1). The postprocessing of these simulation results includes the evaluation of the tissue behaviour by constructing response surfaces. These surfaces give the basis for determining objective functions for the multiobjective optimization (Module $\mathrm{C}$ in Fig. 1). Finally, the optimization algorithm is nested into a large-scale parametric study to provide generally applicable approximate results for the optimal skull opening (Module D in Fig. 1).
In this study, oedematous injury cases are analysed with special emphasis on tissue behaviour during surgery. Three patient-specific models were developed to have validation possibilities by the reconstruction of real-life DC cases (detailed in [12]) and to obtain a large dataset of results and more reliable response surfaces.

Geometry creation starts with the segmentation of the available (injured state) preoperative CT scans of the patients to obtain the outer surface of the brain tissue. In the absence of a generally accepted automatic method [19], a semi-automatic segmentation is applied, where the preliminary results of the threshold-based method are post-processed manually. Since our simulations start from an approximate healthy geometry, CT images of healthy volunteers are applied to obtain the shape of the ventricles (Fig. 2 a, b). It is done by nonlinear medical image registration using B-splines in 3D Slicer environment [20] as it was detailed in previous research [12]. Afterwards, further details of the corpus callosum and the brainstem (which cannot be adequately captured by the CT scans solely) are implemented in the model with the help of the BigBrain Atlas [1] (Fig. 2 a).

In our previous studies [11], [12], complex FE models were validated and applied to perform a couple of simulations with different skull openings. Although these models led to convincing results in some cases, they had two major drawbacks: excessive computational time and convergence problems occur in case of significant ICP (which became the barrier of other research [7] also). Most of these difficulties are caused by the extremely large deformations which result in highly distorted elements near the edges of the skull opening. To perform a lot of simulations with large ICP, a simplified modelling strategy was used in this study.

The appropriate modelling of the pia-arachnoid

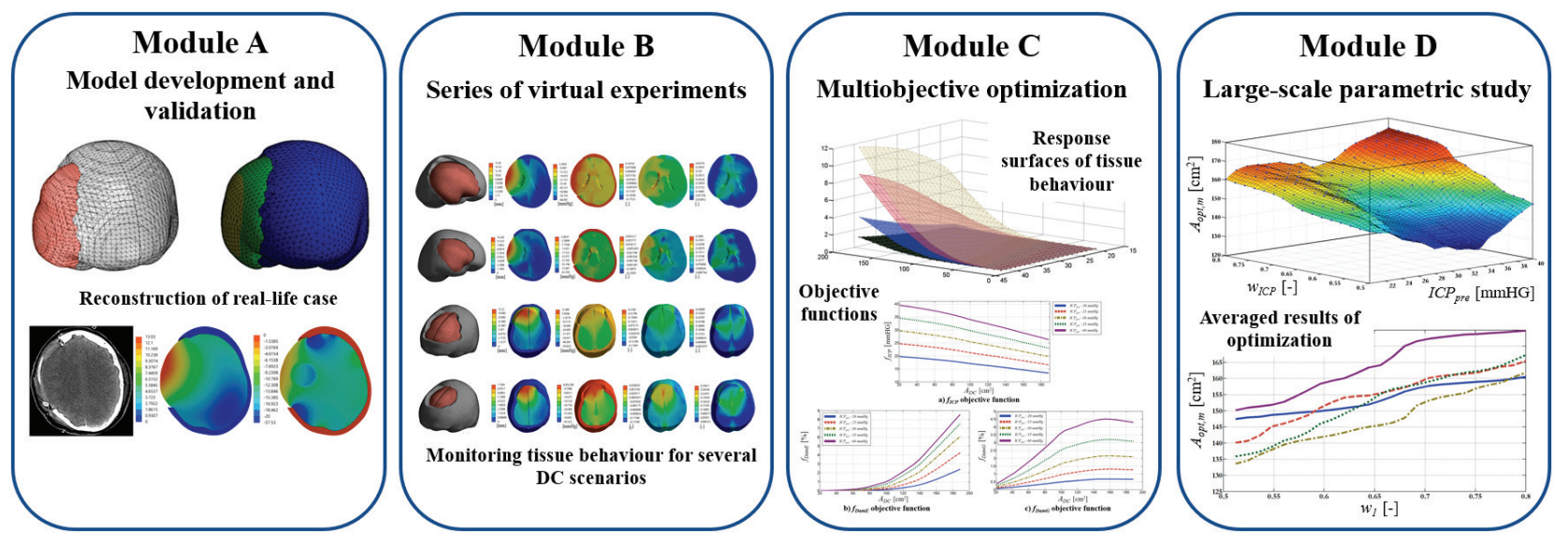

Fig. 1. Modular methodology for the optimization of decompressive craniectomy 
complex has been a key issue during the analysis of TBIs in case of blunt trauma [25] and tumour resection [19], and usually a sliding skull-brain interface is applied. The main motivation behind this choice is that in case of a geometry where the outer CSF spaces are present, significant relative displacements occur between the skull and the brain. However, our current investigations significantly differ from the abovementioned cases, because, due to the swelling of the brain, the outer CSF spaces are compressed. In most of these cases, significant relative displacement cannot occur due to the increased brain volume and the associated compressive forces on the skull-brain interface. The only exception is when such large skull opening is applied that the craniectomy edges are nearly tangential to the tissue. In other cases, significant sliding motion of the brain cannot develop, because it would require the compression of the tissue near the skull opening. During the model selection, this hypothesis was tested by a verification study, in which the results of a previously validated model including a sliding interface were compared to the results of a simplified model where fixed skull-brain interface was applied. A qualitative comparison of the deformation and pressure distributions of the two types of models gave a good agreement while the quantitative comparison of the obtained ICP values revealed only a $3-5 \%$ difference, confirming that in most cases, the boundary conditions have negligible effects in our current field of investigations. Based on this conclusion, our previous models have been simplified by omitting the skull and considering its supporting effect by fixed nodal supports. This simplification enabled the considerable reduction of the computational time and the avoidance of large element distortion at the edges of the skull openings. After this simplification, the models explicitly contain the falx and the brain tissue with the details of the lateral ventricles and the corpus callosum. The ventricles are not modelled by solid domains, but represented as internal cavities. The supporting effects of the CSF are considered by a small pressure load (to approximate a healthy state as detailed below) and soft elastic spring supports (to model that the compression of the ventricles during brain swelling cause pressure increment at the surfaces of the ventricles).

In opposed to previous studies of image-guided neurosurgery [19], where the time-dependent deformations must be captured, we focus on the final (postoperative) deformations only, without the detailed analysis of the time-dependent tissue behaviour. Therefore, following the previous study of DC [7], an isotropic, hyperelastic, second-order Ogden-type material model is applied for the brain based on previous experimental analysis of the slow, large deformations of human brain tissue [9]. The corresponding strain energy function $U$ is shown in Eq. (1):

$$
U=\sum_{i=1}^{2} \frac{2 \mu_{i}}{\alpha_{i}^{2}}\left(\lambda_{1}^{\alpha_{i}}+\lambda_{2}^{\alpha_{i}}+\lambda_{3}^{\alpha_{i}}-3\right),
$$

where:

$\mu_{1}, \mu_{2}-$ parameters which describe the shear modulus,

$\alpha_{1}, \alpha_{2}$ - parameters which characterize the strain hardening,

$\lambda_{1}, \lambda_{2}, \lambda_{3}-$ deviatoric principal stretches.

The falx is modelled by a linear elastic material model. The value of the material parameters is summarized in Table 1 (where $E$ is the Young's modulus and $v$ is the Poisson's ratio).

The FE models are developed in Ansys Workbench [2], where with the application of the so-called patch-independent algorithm, a high-quality tetrahedral mesh could be generated even for the highly irregular geometry of the brain. The convergence analysis confirmed that with our current meshes containing 120,000-170,000 elements (depending on the opening size due to the local refinement near its edges) the required level of accuracy is reached. To avoid volumetric locking, the mesh contains special fournode tetrahedral elements where the pressure is an extra degree of freedom at each node. These elements are associated with pressure stabilization and they are used with a mixed u-P formulation [2]. For the falx, a quadrilateral shell FE mesh was generated (Fig. $2 \mathrm{c}$ ).

The currently analysed quasi-static cases of brain swelling and DC are modelled by static simulations with implicit time stepping in Ansys [2]. Large deformations are expected, thus geometric nonlinearities are considered by the Updated Lagrangian formulation. Each simulation includes three load steps. Firstly, a $5 \mathrm{mmHg}$ pressure is applied to the initial (stress--free) geometry of the brain, to reach an approximate healthy state. In the second load step, the swelling of the brain is modelled by a uniformly distributed fictive thermal expansion of the tissue to reach the preoperative (i.e., injured) state. In the third load step, the DC is performed by deleting the nodal constraints at the skull opening to reach the postoperative state.

Simulation series are performed for six different preoperative pressure $\left(I C P_{\text {pre }}\right)$ values (ranging from $16.5 \mathrm{mmHg}$ to $41.5 \mathrm{mmHg}$ ) with 10 unilateral (ranging from $20 \mathrm{~cm}^{2}$ to $200 \mathrm{~cm}^{2}$ ) and 10 bifrontal (ranging from $10 \mathrm{~cm}^{2}$ to $100 \mathrm{~cm}^{2}$ ) skull openings areas $\left(A_{D C}\right)$. 
Table 1. Applied material models and their parameters

\begin{tabular}{|c|c|c|c|c|}
\hline Anatomic part & Material model & \multicolumn{3}{|c|}{ Material parameters } \\
\hline Dura mater and falx cerebri & Linear elastic & $E=31.5 \mathrm{MPa}$ & $v=0.45$ & \\
\hline \multirow{2}{*}{ Brain tissue } & Hyperelastic & $\mu_{1}=1.044 \mathrm{kPa}$ & $\alpha_{1}=4.308$ & \multirow{2}{*}{$D_{1}=D_{2}=3 \cdot 10-4 \frac{1}{\mathrm{~Pa}}$} \\
\hline & 2nd order Ogden & $\mu_{2}=1.183 \mathrm{kPa}$ & $\alpha_{2}=7.736$ & \\
\hline
\end{tabular}

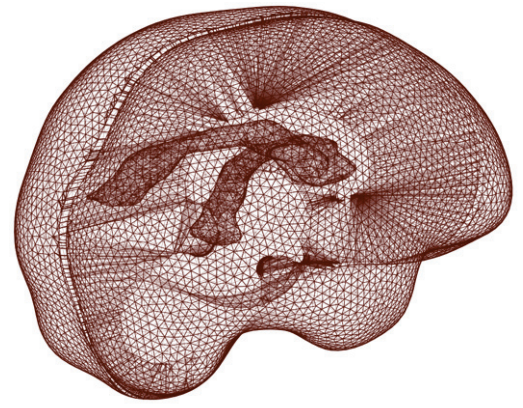

a) Wireframe view of brain geometry

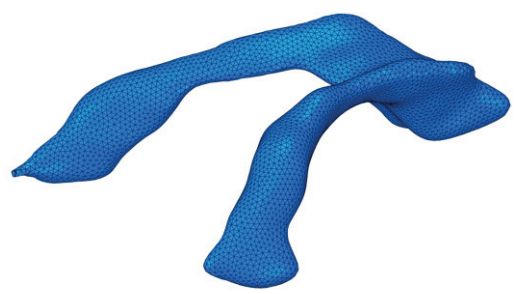

b) Geometry of lateral ventricles

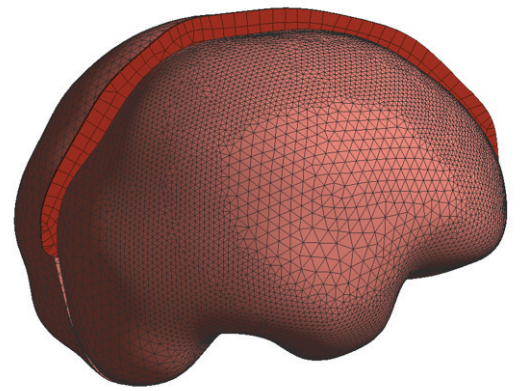

c) FE mesh of the brain and falx

Fig. 2. Geometry and mesh of a FE model: (a) wireframe view of the brain geometry, (b) geometry of lateral ventricles, (c) FE mesh of the brain and falx
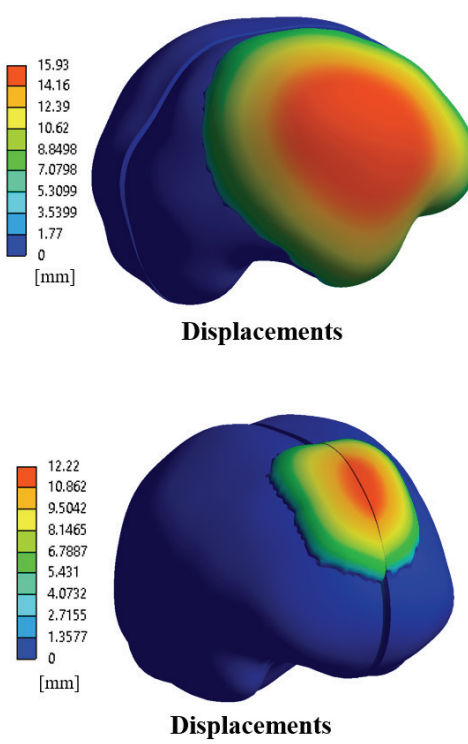

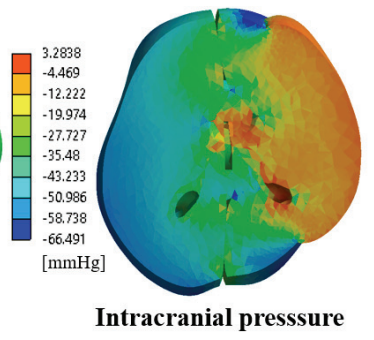

cranial presssure

a) FE analysis of

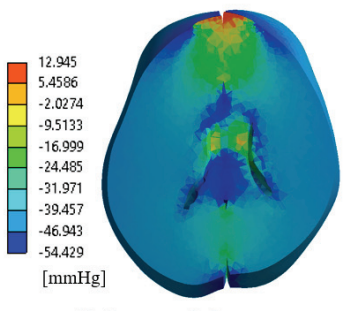

Intracranial presssure

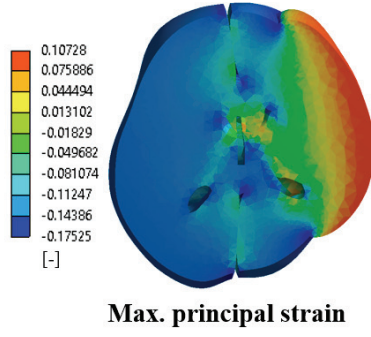

Max. principal strain

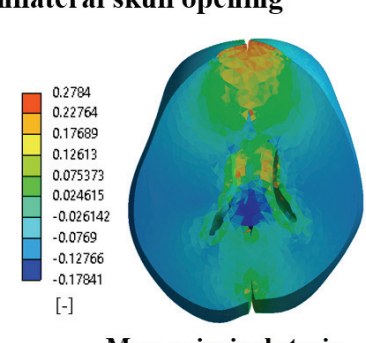

Max. principal strain

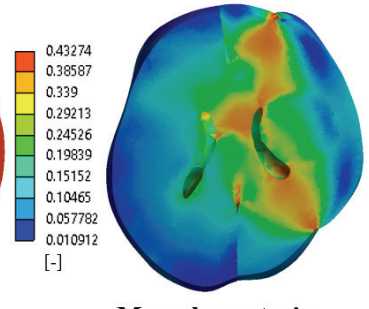

Max. shear strain

b) FE analysis of bifrontal skull opening

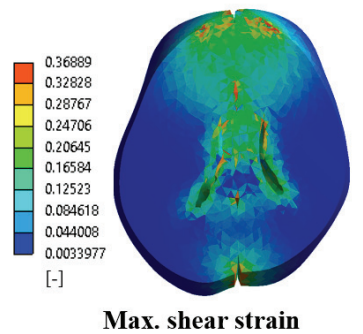

Max. shear strain

Fig. 3. FE simulation of decompressive craniectomy: (a) FE analysis of unilateral skull opening, (b) FE analysis of bifrontal skull opening

The mathematical formulation of the optimization problem is based on the monitoring of three output measures (Fig. 3) which provides the basis for the construction of objective functions. One key output parameter of the FE simulations is the postoperative pressure $\left(I C P_{\text {post }}\right)$ (Fig. 3). Since currently, the gold standard of ICP measurement is performed in one of the ventricles using the External Ventricular Drainage 
technique, $I C P_{\text {post }}$ should be evaluated near the ventricles also. Instead of monitoring ICP at one point only (where the results would be sensitive to the applied locations of the measurement), $I C P_{\text {post }}$ is quantified by taking the average of the ICP over elements which belong to a $4-4.5 \mathrm{~cm}$ radius sphere (depending on the size of the current model) at the centre of the brain. The other two outputs being monitored are intended to describe the risk of functional damage due to the tissue deformations during surgery. In this study, two damage mechanisms are considered. One of them is related to the shear distortion of the tissue (Fig. 3), which is thought to be a key factor in the development of injury, especially since previous DC studies pointed out that large shear strains tend to occur along the perimeter of the skull opening and near the ventricles [12], [24]. The risk of damage associated with shear is quantified by the relative brain volume $\left(V_{\text {DamG }}\right)$, where the maximum shear strain exceeds a threshold value $\left(T_{\text {DamG }}\right)$. Based on previous biomechanical study of DC [7], the application of a $T_{\text {DamG }}=0.35$ threshold may be reasonable, however, tissue deformations are usually maintained for a much longer time after surgery than it was analysed by the dynamic tests of organotypic slice cultures of rat cortex [4]. Therefore, 0.35 is considered as an upper bound in this study, and the $T_{\text {DamG }}=0.15-0.35$ threshold interval is analysed during the large-scale parametric study. The other damage mechanism being considered is associated with the extreme stretches of the tissue due to the bulging displacement (Fig. 3), and it is quantified by maximum principal strains (MPS). Usually, the observed magnitude of MPS is smaller than the maximum shear strains. However, based on the analysis of the sciatic nerve using stretching models, it has been concluded that even minimal tension can lead to neurological damage if it is maintained for a relatively long time [8]. Therefore, it is expected that the damage to the central nervous system can also occur due to long-duration stretches [24]. Despite the lack of specific knowledge of this threshold $\left(T_{\text {DamE }}\right)$, a $T_{\text {DamE }}=$ $0.025-0.1$ interval is considered during the large-scale parametric study. Accordingly, the optimization is based on the minimization of three objective functions, which are functions of the skull opening area $\left(A_{D C}\right)$ : postoperative ICP $\left(f_{\mathrm{ICP}}\right)$, damaged relative brain volume due to shear strain $\left(f_{\text {DamG }}\right)$ and damaged relative brain volume due to MPS $\left(f_{\text {DamE }}\right)$. These objectives functions are obtained in two main steps: (1) a response surface of the mentioned output parameters (in the function of the preoperative pressure and the skull opening area) is fitted to the FEM results by cubic spline interpolation and (2) the objective func- tion associated with a certain preoperative ICP is cut out from the response surface.

This multiobjective optimization problem is solved by scalarization using two different methods based on the formulation of one resultant objective function using relative weights, exponents and penalty parameters. The first scalarization method is called Goal Programming (GP) method [18] where the resultant objective function $\left(F_{\mathrm{GP}, i}\right)$ has the following form:

$$
\begin{aligned}
F_{\mathrm{GP}, i}=w_{\mathrm{ICP}}( & \left.\frac{f_{\mathrm{ICP}}-g_{\mathrm{ICP}}}{b_{\mathrm{ICP}}-g_{\mathrm{ICP}}}\right)^{n_{i}}+w_{\text {DamE }}\left(\frac{f_{\text {DamE }}-g_{\text {DamE }}}{b_{\text {DamE }}-g_{\text {DamE }}}\right)^{n_{i}} \\
& +w_{\text {DamG }}\left(\frac{f_{\text {DamG }}-g_{\text {DamG }}}{b_{\text {DamG }}-g_{\text {DamG }}}\right)^{n_{i}}
\end{aligned}
$$

where:

$f_{\mathrm{ICP}}, f_{\text {DamE }}, f_{\text {DamG }}-$ objective functions,

$w_{\text {ICP }}, w_{\text {DamE }}, w_{\text {DamG }}-$ relative weights of the objective functions,

$g_{\text {ICP }}, g_{\text {DamE }}, g_{\text {DamG }}-$ "good" values of the objective functions,

$b_{\text {ICP }}, b_{\text {DamE }}, b_{\text {DamG }}$ - "bad" values of the objective functions,

$n_{i}$ - exponent.

In this study, each individual optimization is repeated for two different resultant objective functions constructed with the GP method $\left(F_{\mathrm{GP}, 1}\right.$ and $\left.F_{\mathrm{GP}, 2}\right)$ using $n_{1}=1$ and $n_{2}=3$, respectively. During the large-scale parametric investigations, the $w_{\text {ICP }}=0.5-0.8$ interval is analysed with the constraints: $w_{\text {DamE }}=w_{\text {DamG }}$ and $w_{\text {ICP }}+w_{\text {DamE }}+w_{\text {DamG }}=1$. The "good" and "bad" values of the objective functions are taken in the following way: $g_{\mathrm{ICP}}=15 \mathrm{mmHg}, b_{\mathrm{ICP}}=25 \mathrm{mmHg}, g_{\mathrm{DamE}}=$ $g_{\text {DamG }}=1 \%, b_{\text {DamE }}=b_{\text {DamG }}=5 \%$.

The other applied scalarization technique is the "so-called" Physical Programming (PP) method [18], where a penalty function is associated with each objective function. The currently applied penalty functions (Fig. 4) are constructed by linear interpolation to point pairs summarized in Table 2 .

The resultant objective functions with the PP method are constructed from the penalty functions in the following way:

$$
F_{\mathrm{PP}, i}=w_{\mathrm{ICP}} P_{\mathrm{ICP}}^{n_{i}}+w_{\text {DamE }} P_{\text {DamE }}^{n_{i}}+w_{\text {DamG }} P_{\text {DamG }}^{n_{i}},
$$

where:

$w_{\text {ICP }}, w_{\text {DamE }}, w_{\text {DamG }}-$ relative weights of the objective functions,

$P_{\mathrm{ICP}}, P_{\text {DamE }}, P_{\text {DamG }}-$ penalty functions,

$n_{i}$ - exponent. 


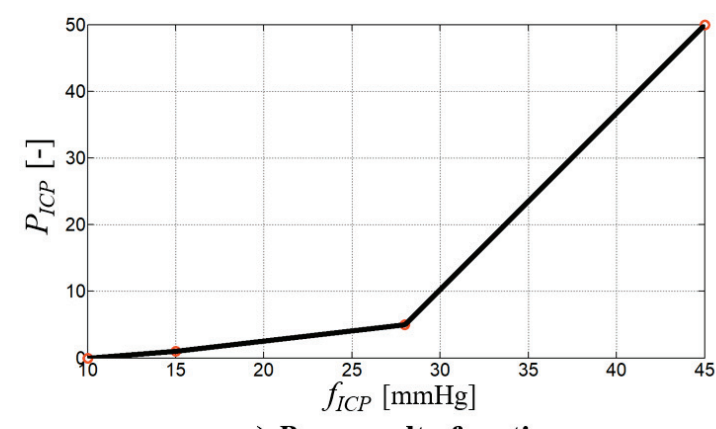

a) $P_{I C P}$ penalty function

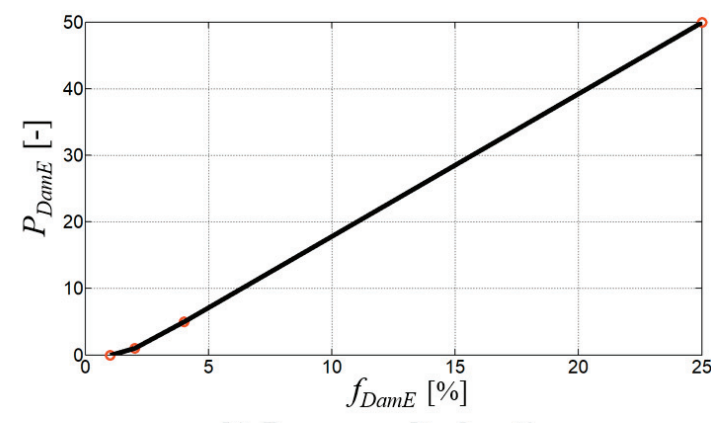

b) $\boldsymbol{P}_{\text {DamE }}$ penalty function

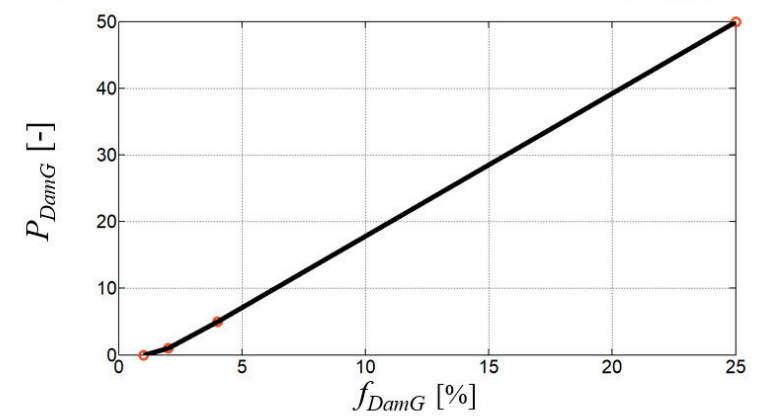

c) $\boldsymbol{P}_{\text {DamG }}$ penalty function

Fig. 4. Penalty functions for scalarization with the Physical Programming method:

(a) $P_{\text {ICP }}$ penalty function, (b) $P_{\text {DamE }}$ penalty function, (c) $P_{\text {DamG }}$ penalty function

Table 2. Point pairs which define the multilinear penalty functions

\begin{tabular}{|c|c|c|c|c|c|}
\hline$f_{\mathrm{ICP}}$ & $P_{\text {ICP }}$ & $f_{\text {DamE }}$ & $P_{\text {DamE }}$ & $f_{\text {DamG }}$ & $P_{\text {DamG }}$ \\
\hline 10 & 0 & 1 & 0 & 1 & 0 \\
\hline 15 & 1 & 2 & 1 & 2 & 1 \\
\hline 28 & 5 & 4 & 5 & 4 & 5 \\
\hline 45 & 50 & 25 & 50 & 25 & 50 \\
\hline
\end{tabular}

Similarly to the GP method, each optimization is repeated for two different resultant objective functions constructed with the PP method $\left(F_{\mathrm{PP}, 1}\right.$ and $\left.F_{\mathrm{PP}, 2}\right)$ using $n_{1}=1$ and $n_{2}=2$, respectively. In this paper, the optimal skull opening of one individual optimization problem $\left(A_{\text {opt }}\right)$ is considered to be the mean value of the optimal openings obtained by the mentioned four resultant objective functions.

The mathematical formulation of the optimization problem has been implemented in Matlab [22], and this algorithm enabled us to determine $A_{\text {opt }}$ in the case where all of the input parameters (patient-specific FE results, $I C P_{\text {post }}$, relative weights, resistance thresholds etc.) are known. Currently, it would be very optimistic to believe that all of these parameters would be available in the close future at the time of the decisionmaking. Therefore, generally applicable approximate results are generated based on the currently available FE results. To obtain this purpose, the above-detailed optimization strategy was nested into a large-scale parametric study, and the $A_{\text {opt }}$ results were averaged over the previously mentioned intervals of $T_{\mathrm{DamE}}$ and $T_{\text {DamG }}$.

\section{Results}

Based on the results of a large number of FE simulations, response surfaces of the FE output parameters have been determined. In Figure 5, response surfaces corresponding to unilateral skull openings are shown, while the observed tendencies are similar for bifrontal skull openings as well. These surfaces indicate how tissue behaviour depends on $I C P_{\text {pre }}$ and $A_{D C}$, and these are used to construct objective function. In Figure 5 a, it can be seen how $I C P_{\text {post }}$ decreases as $A_{D C}$ increases and $I C P_{\text {pre }}$ decreases. In Figures $5 \mathrm{~b}, \mathrm{c}$, the relative volume of the damaged tissue $\left(V_{\mathrm{DamE}}\right.$ and $\left.V_{\text {DamG }}\right)$ are shown for several threshold values ( $T_{\text {DamE }}$ and $\left.T_{\text {DamG }}\right)$. It can be observed how large damage tends to occur as $A_{D C}$ and $I C P_{\text {pre }}$ increases and how it depends on the applied thresholds. It is noted that response surfaces in Fig. 5 do not belong to one particular FE model, but these are resultant averaged surfaces of all FE simulations series performed by three different models. The construction of these surfaces includes two main steps: (1) scaling the patientspecific FE results to an averaged geometry (which had a $600 \mathrm{~cm}^{2}$ large brain surface) based on the ratios 


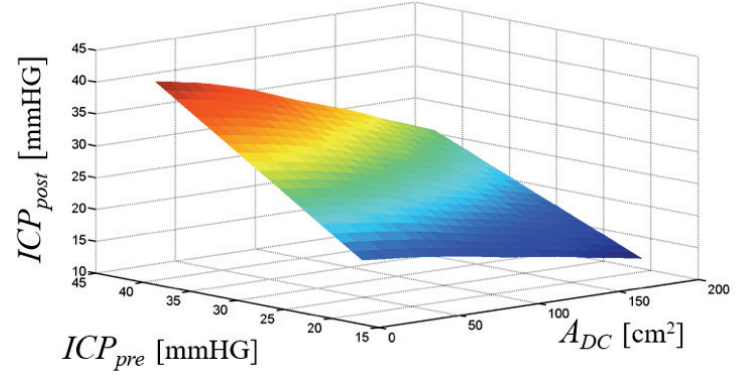

a) $I C P_{p o s t}$ response surface

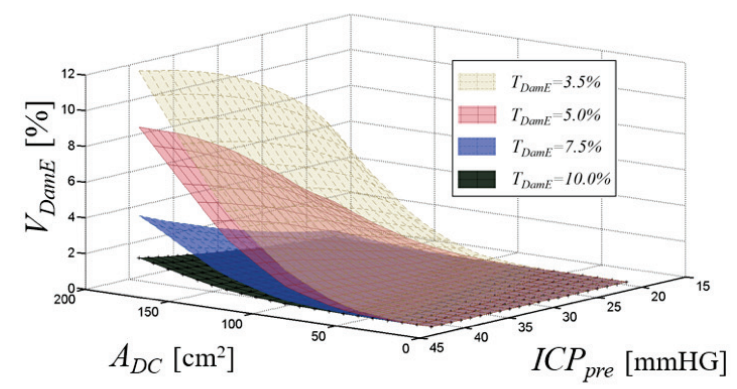

b) $V_{\text {DamE }}$ response surface

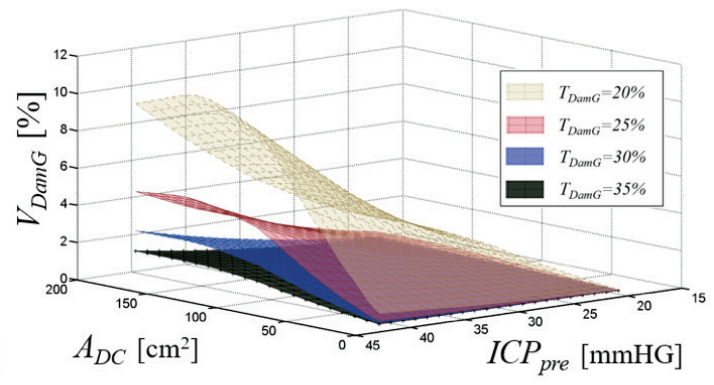

c) $V_{D a m G}$ response surface

Fig. 5. Response surfaces of the brain tissue in terms of the monitored outputs: (a) $I C P_{\text {post }}$ response surface, (b) $V_{\text {DamE }}$ response surface, (c) $V_{\text {DamG }}$ response surface

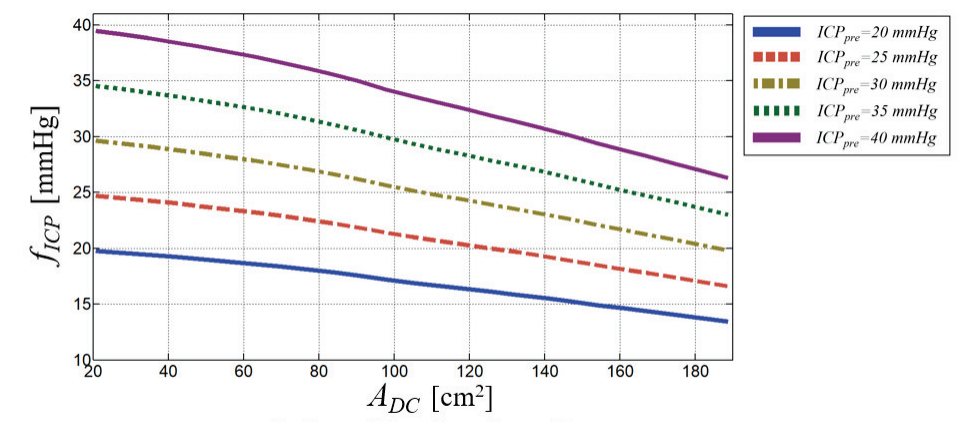

a) $f_{I C P}$ objective function

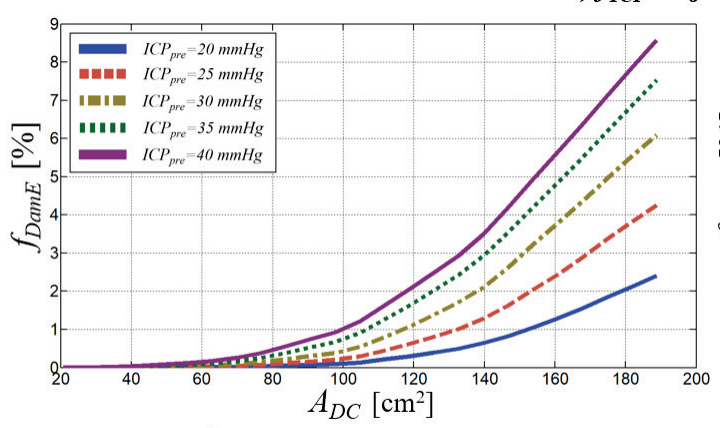

b) $f_{\text {DamE }}$ objective function

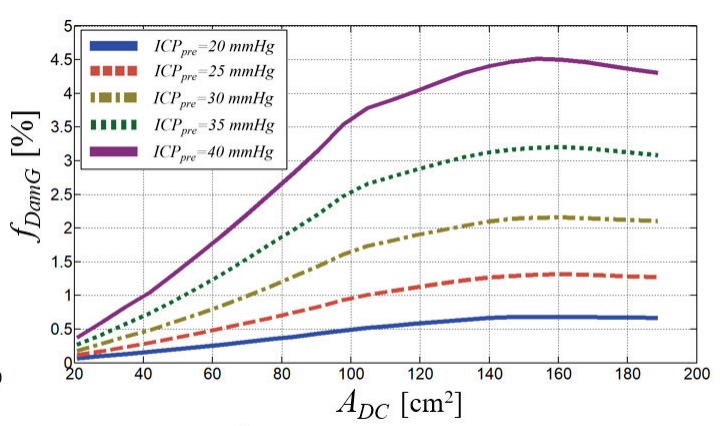

c) $f_{\text {DamG }}$ objective function

Fig. 6. Objective functions for unilateral DC with the assumption of $T_{\mathrm{DamE}}=0.05$ and $T_{\mathrm{DamG}}=0.25$ :

(a) $f_{\text {ICP }}$ objective function, (b) $f_{\text {DamE }}$ objective function, (c) $f_{\text {DamG }}$ objective function

of the outer brain surfaces and (2) taking the averages of the scaled patient-specific surfaces. Patient-specific surfaces could be used where the task is the optimization of DC for one particular patient. However, this scaling and averaging concept may fit better to the goal of this paper where $A_{\text {opt }}$ results are presented in a more generally applicable way.

It is assumed that $I C P_{\text {pre }}$ is known at each individual optimization problem since it can be measured at the clinics by several methods. Accordingly, the ob- 
jective functions of the optimization can be cut out of the response surfaces at a given $I C P_{\text {pre }}$ value. In Figure 6 , objective functions are shown for several $I C P_{\text {pre }}$ value obtained by the $T_{\mathrm{DamE}}=0.05$ and $T_{\mathrm{DamG}}=0.25$ assumption.

Once the objective functions were constructed, the individual optimization for a given $I C P_{\text {pre }}$ could be performed by setting the $w_{\text {ICP }}$ weight. Results of the in- dividual optimization cases are summarized in Table 3 for several $w_{\text {ICP }}$ values (with the $T_{\text {DamE }}=0.05$ and $T_{\text {DamG }}=0.25$ assumption) for unilateral and bifrontal skull openings as well.

In Table 3, the mean value of the four resultant objective functions $\left(P_{m}\right)$ at the optimal skull opening is also shown. This enables us to compare the efficiency of the optimal unilateral and bifrontal skull

Table 3. Optimization results for several $w_{\mathrm{ICP}}$ and $I C P_{\text {pre }}$ input pairs with the assumption of $T_{\mathrm{DamE}}=0.05$ and $T_{\text {DamG }}=0.25$ ( ${ }^{*}$ indicates that the current result is governed by the upped bound of the optimization)

\begin{tabular}{|c|c|c|c|c|c|c|c|}
\hline \multirow{3}{*}{$\begin{array}{c}I C P_{\text {pre }} \\
{[\mathrm{mmHg}]}\end{array}$} & & \multicolumn{6}{|c|}{$w_{\text {ICP }}[-]$} \\
\hline & & \multicolumn{2}{|c|}{0.6} & \multicolumn{2}{|c|}{0.7} & \multicolumn{2}{|c|}{0.8} \\
\hline & & $\begin{array}{c}A_{\mathrm{opt}} \\
{\left[\mathrm{cm}^{2}\right]}\end{array}$ & $\begin{array}{l}P_{m} \\
{[-]}\end{array}$ & $\begin{array}{c}A_{\mathrm{opt}} \\
{\left[\mathrm{cm}^{2}\right]}\end{array}$ & $\begin{array}{l}P_{m} \\
{[-]}\end{array}$ & $\begin{array}{c}A_{\mathrm{opt}} \\
{\left[\mathrm{cm}^{2}\right]}\end{array}$ & $\begin{array}{l}P_{m} \\
{[-]}\end{array}$ \\
\hline \multirow{2}{*}{20} & Unilateral & 156.9 & 0.254 & 165.0 & 0.280 & 169.5 & 0.284 \\
\hline & Bifrontal & $90^{*}$ & $0.636^{*}$ & $90^{*}$ & $0.750^{*}$ & $90^{*}$ & $0.863^{*}$ \\
\hline \multirow{2}{*}{25} & Unilateral & 155.2 & 1.10 & 164.1 & 1.22 & 171.5 & 1.29 \\
\hline & Bifrontal & $90^{*}$ & $1.80^{*}$ & $90^{*}$ & $2.10^{*}$ & $90^{*}$ & $2.41^{*}$ \\
\hline \multirow{2}{*}{30} & Unilateral & 141.7 & 2.64 & 157.2 & 2.95 & 168.7 & 3.19 \\
\hline & Bifrontal & $90^{*}$ & $3.56^{*}$ & $90^{*}$ & $4.16^{*}$ & $90^{*}$ & $4.76^{*}$ \\
\hline \multirow{2}{*}{35} & Unilateral & 136.9 & 5.13 & 153.3 & 5.61 & 159.3 & 5.97 \\
\hline & Bifrontal & $90^{*}$ & $14.7^{*}$ & $90^{*}$ & $17.1^{*}$ & $90^{*}$ & $19.6^{*}$ \\
\hline \multirow{2}{*}{40} & Unilateral & 158.3 & 12.0 & 172.0 & 11.1 & 174.3 & 10.1 \\
\hline & Bifrontal & $90^{*}$ & $67.4^{*}$ & $90^{*}$ & $78.6^{*}$ & $90^{*}$ & $89.9^{*}$ \\
\hline
\end{tabular}

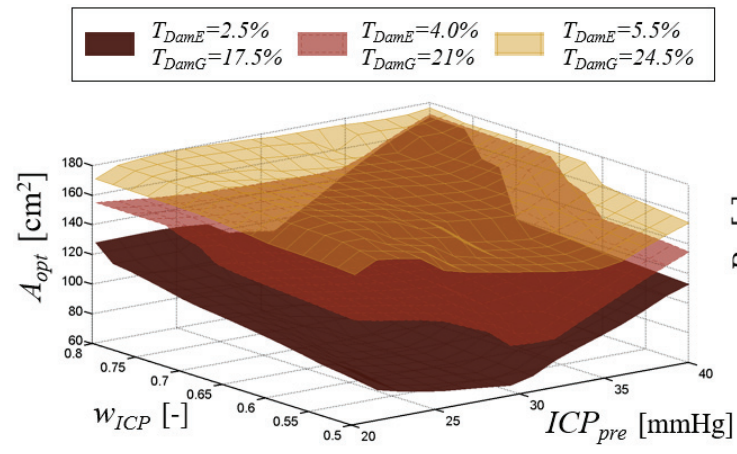

a) Optimal unilateral skull opening

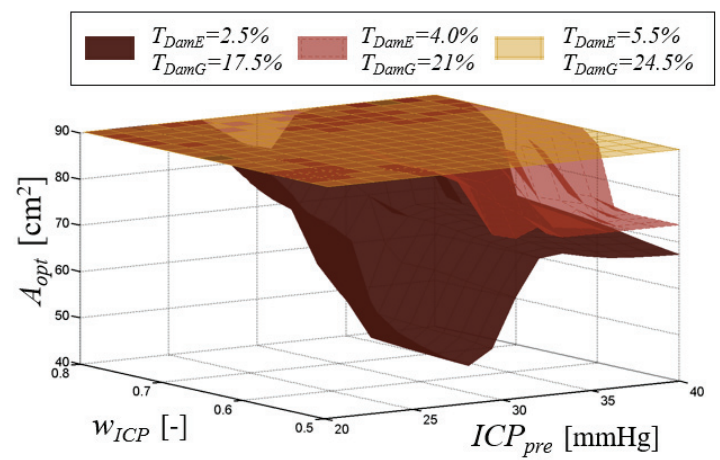

c) Optimal bifrontal skull opening

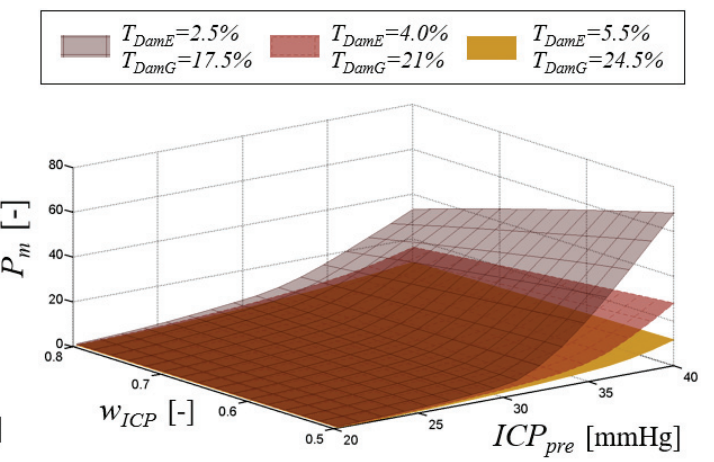

b) $\boldsymbol{P}_{m}$ for unilateral skull opening

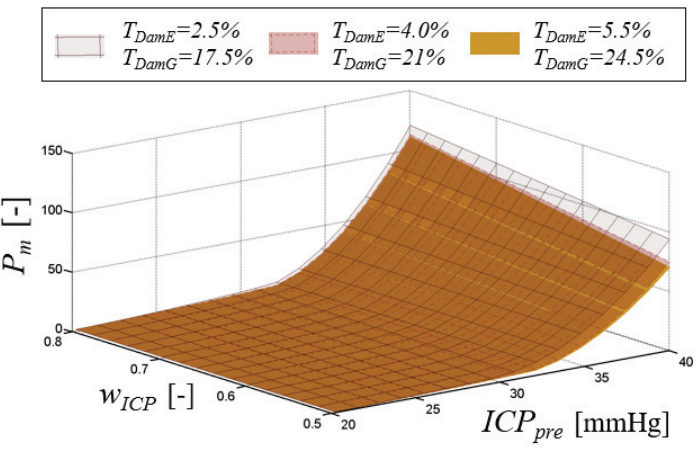

d) $\boldsymbol{P}_{m}$ for bifrontal skull opening

Fig. 7. Dependence of the optimization results on the applied thresholds: (a) optimal unilateral skull opening, (b) $P_{m}$ for unilateral opening, (c) optimal bifrontal skull opening, (d) $P_{m}$ for bifrontal opening 

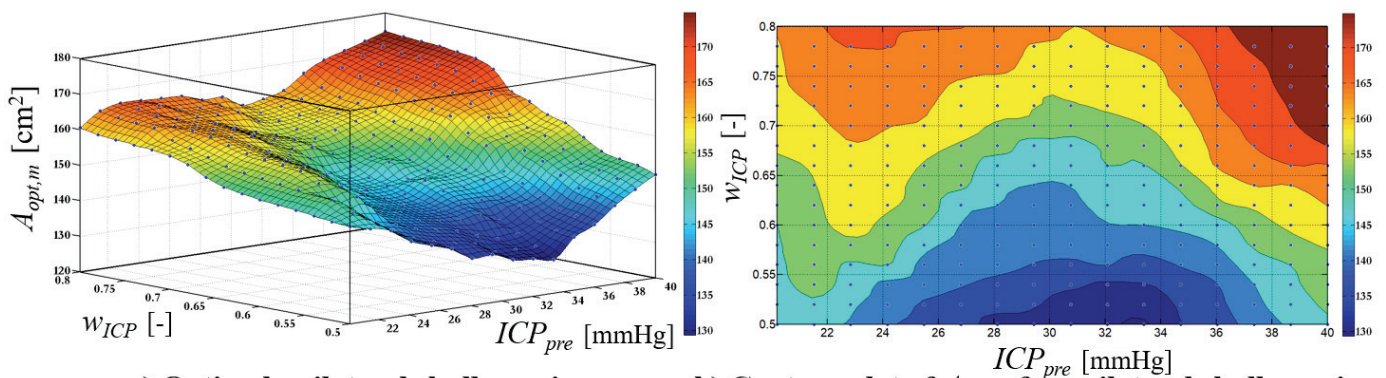

a) Optimal unilateral skull opening

b) Contour plot of $A_{o p t, m}$ for unilateral skull opening
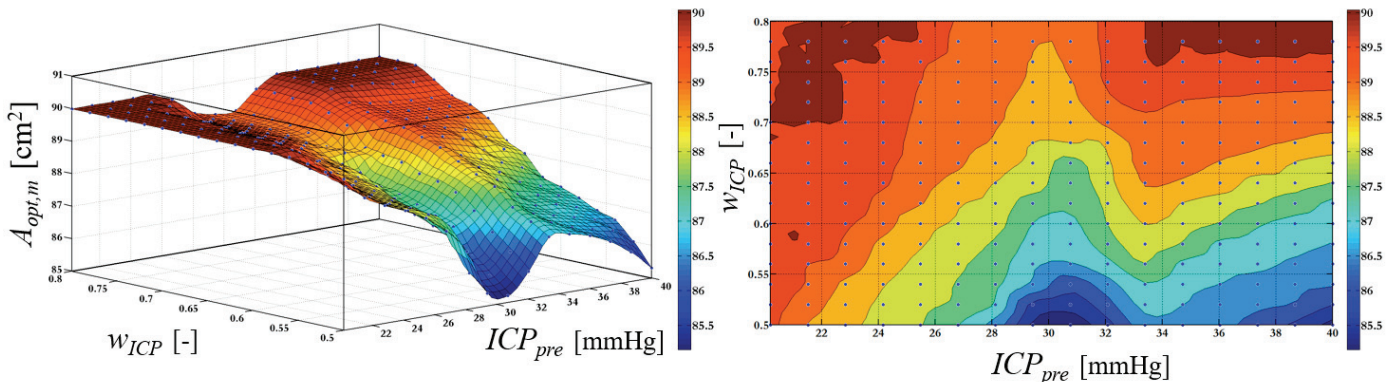

c) Optimal bifrontal skull opening

d) Contour plot of $A_{o p t, m}$ for bifrontal skull opening

Fig. 8. Averaged optimal skull opening based on large-scale parametric study: (a) optimal unilateral skull opening, (b) contour plot of $A_{\mathrm{opt}, m}$ for unilateral opening, (c) optimal bifrontal skull opening, (d) contour plot of $A_{\mathrm{opt}, m}$ for bifrontal opening

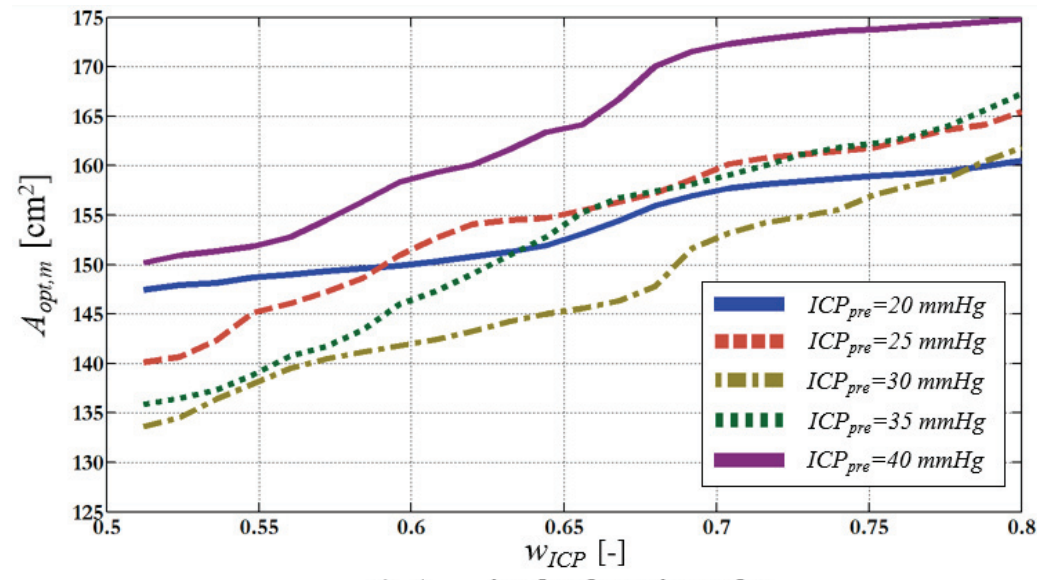

a) $A_{o p t, m}$ in the function of $w_{1}$

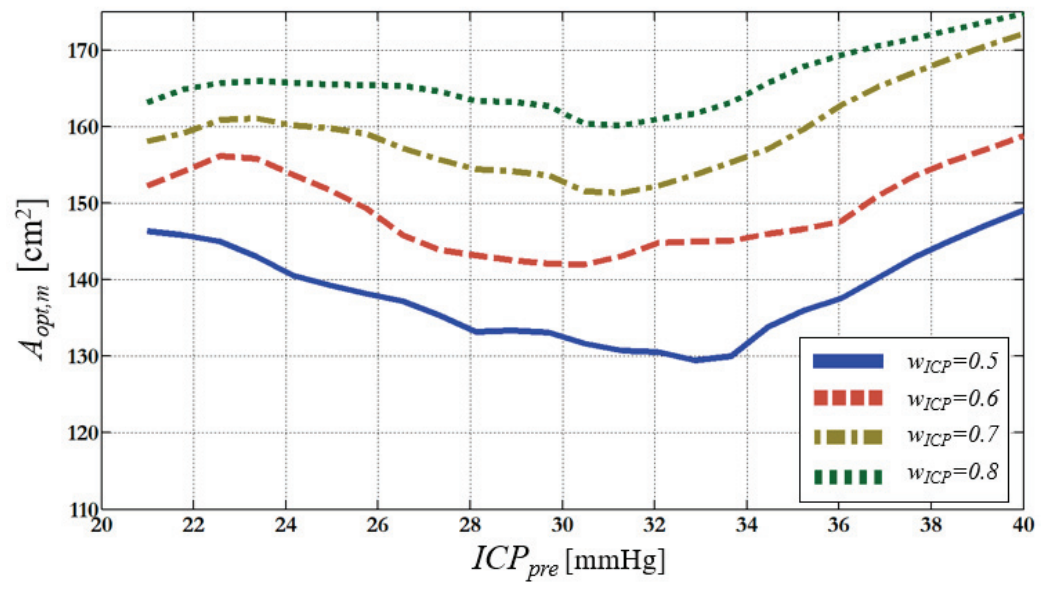

b) $A_{o p t, m}$ in the function of $I C P_{p r e}$

Fig. 9. Set of curves of averaged optimal craniectomy size for unilateral skull opening based on large-scale parametric study: (a) $A_{\mathrm{opt}, m}$ as the function of $w_{\mathrm{ICP}}$, (b) $A_{\mathrm{opt}, m}$ in the function of $I C P_{\mathrm{pre}}$ 
openings. It is noted that the optimization algorithm has run between bounds of $A_{D C}=60-190 \mathrm{~cm}^{2}$ for the unilateral and $A_{D C}=20-90 \mathrm{~cm}^{2}$ for the bifrontal skull opening. Accordingly, it can be observed that with the current input parameters the optimal bifrontal skull opening is always equalled to the upper bound of the optimization. Furthermore, in all of these cases, $P_{m}$ is smaller for the unilateral skull opening which predicts that the unilateral opening is much more favourable than the currently analysed bifrontal craniectomy. It is important to emphasize that the presented results of the individual optimization (Table 3) depend on the applied $T_{\text {DamE }}$ and $T_{\text {DamG }}$ thresholds, but, in general, as these thresholds increase, $A_{\text {opt }}$ increases while $P_{m}$ decreases. This dependence is illustrated in Fig. 7 for three different threshold pairs.

Since currently the exact value of these thresholds are not readily available, the optimal skull opening during the large-scale parametric study $\left(A_{\mathrm{opt}, m}\right)$ is determined by averaging the results of individual optimization over the analysed interval of the thresholds. The surface of the optimal skull opening $\left(A_{\mathrm{opt}, m}\right)$ as the function of $I C P_{\text {pre }}$ and $w_{\text {ICP }}$ is shown in Figs. $8 \mathrm{a}-\mathrm{b}$ for the unilateral and in Figs. $8 \mathrm{c}-\mathrm{d}$ for bifrontal skull openings.

For unilateral skull openings, which seemed to be more efficient than the bifrontal opening (Table 3), the obtained $A_{\mathrm{opt}, m}$ results are also illustrated by the set of curves which may be applicable more easily at the time of the decision-making. In Figure $9 \mathrm{a}, A_{\mathrm{opt}, m}$ is shown as the function of the $w_{\text {ICP }}$ weight for different $I C P_{\text {pre }}$ values, while in Figure $9 \mathrm{~b}, A_{\mathrm{opt}, m}$ is shown as the function of the $I C P_{\text {pre }}$ for different $w_{\text {ICP }}$ parameters.

\section{Discussion}

In this paper, the engineering optimization of DC has been performed for oedematous cases from a biomechanical perspective based on the results of numerical simulations. As a first step, a large number of FE simulations have been carried out to perform the comprehensive analysis of the biomechanical behaviour of the brain tissue during surgery. These results (Fig. 5) give insight into how the tissue response depends on the preoperative pressure, the location and the size of the skull opening and tissue resistance. Afterwards, the formulation of the mathematical background of the optimization problem has been completed. Objective functions have been identified corresponding to possible damage mechanisms and several resultant objec- tive functions have been constructed (Eqs. (2), (3)) to cope with the multiobjective optimization. The optimization has been performed for several individual cases with certain realizations of input parameters (Table 3). Some tendencies have been also shown to illustrate how $A_{\text {opt }}$ depends on these input parameters (Fig. 7). Finally, more generally applicable approximate results have been determined based on a largescale parametric study, which may help at the decision-making procedure during the design of the craniectomy (Figs. 8 and 9).

In Figure 5 a, it is shown how $I C P_{\text {post }}$ depends on $I C P_{\text {pre }}$ and $A_{D C}$. It could be observed that the $I C P_{\text {post }}$ has an approximately linear dependence not just on the skull opening area (which has been shown in a previous study [11]), but on the preoperative pressure as well. Accordingly, a plane could be fitted to the response surface in Fig. 5 a, which will result in an easily applicable method for the design of surgery. On the other hand, the relative volume of the damaged brain tissue depends on $I C P_{\text {pre }}$ and $A_{D C}$ in a more general nonlinear manner, as shown in Figs. 5b, c. Based on the results of a parametric study, it is also shown how the extent of the damage depends on the applied thresholds of the tissue resistance (Figs. 5b, c). Unfortunately, these thresholds are not readily available currently. Therefore, the results of the individual optimization cases are shown for several $T_{\text {DamE }}$ and $T_{\text {DamG }}$ pairs of input parameters. Objective functions of the optimization problem are cut out of the response surfaces and these are illustrated in Fig. 6 (with the assumption of $T_{\text {DamE }}=0.05$ and $T_{\text {DamG }}=0.25$ ) for several $I C P_{\text {pre }}$ values. These curves can give some guidance about how large pressure reduction (Fig. 6a) and damage (Figs. 6b, c) can be anticipated before the execution of DC. Unlike the $T_{\text {DamE }}$ and $T_{\text {DamG }}$ thresholds, $w_{\text {ICP }}$ is an input parameter which should be set by neurosurgeons based on the relative importance of the pressure reduction. In Table $3, A_{\text {opt }}$ results are summarized with the assumption for several pairs of $w_{\text {ICP }}$ and $I C P_{\text {pre }}$ inputs. One major conclusion of these results is that the optimal unilateral skull opening usually falls within the $130-180 \mathrm{~cm}^{2}$ interval. This observation is in good agreement with current medicine recommendations, where a minimum $12 \mathrm{~cm}$ diameter is suggested for a craniectomy. However, for bifrontal skull openings, $A_{\text {opt }}$ in Table 3 equals the upper bound $\left(90 \mathrm{~cm}^{2}\right)$ of the considered $A_{D C}$ interval for all of the analysed cases. It indicates that the real optimum typically belongs to an even larger skull opening area, whose determination could be the topic of further research. Based on the comparison of the obtained $P_{m}$ values (Table 3), it can be stated that the 
unilateral skull opening is more beneficial than the currently considered bifrontal openings. It should be noted that this may be the case only for these oedematous injuries and for this type of biomechanical formulation of the optimization problem. It is not stated that for other injury types or due to other relevant medical aspects the bifrontal opening can not necessarily be an optimal solution. Since results in Table 3 are also based on the $T_{\mathrm{DamE}}=0.05$ and $T_{\mathrm{DamG}}=$ 0.25 assumption, individual optimization has been repeated for several other threshold pairs, and the obtained tendencies are illustrated in Fig. 7. In Figures 7a, c, it can be observed how $A_{\text {opt }}$ increases when larger resistance thresholds are considered, while Figs. 7b, d show how $P_{m}$ increases nonlinearly due to the increment of $I C P_{\text {pre. }}$.

After performing several individual optimizations, its algorithm has been nested into a large-scale parametric study to obtain approximate, generally applicable results. To eliminate the fact that each $A_{\text {opt }}$ result depends on the applied thresholds, the concept of the $A_{\text {opt }, m}$ has been implemented which is obtained by averaging $A_{\text {opt }}$ results over the considered interval of $T_{\text {DamE }}$ and $T_{\text {DamG }}$. The obtained surface of $A_{\mathrm{opt}, m}$ results is shown in Figs. 8a, b for unilateral and in Figs. 8c, d for bifrontal skull opening. Based on these results, the optimal unilateral skull opening area seems to belong to the $130-180 \mathrm{~cm}^{2}$ interval, while for bifrontal skull opening the bulk of these results tend to be equal to the considered upper bound of the optimization. The dependence of $A_{\mathrm{opt}, m}$ on the $w_{\mathrm{ICP}}$ and $I C P_{\mathrm{pre}}$ inputs are further illustrated by the contour plots of Figs. 8b, d. These results are also illustrated by the set of curves (Fig. 9) which provides more easily applicable guidance on how the optimal skull opening area depends on the preoperative pressure and the relative importance of ICP reduction. In Figure $9 \mathrm{~b}$, an increasing tendency of $A_{\mathrm{opt}, m}$ could be observed for relatively large pressure values, indicating that the pressurerelated objective function becomes more and more dominant above 32-34 mmHg ICP pre values.

Most probably, the decision making should depend on several other medical circumstances whose consideration was out of the scope of this paper. Therefore, current observations are strictly the results of the engineering optimization methodology, which was performed by taking the emphasize on the biomechanical perspective of the problem. Hence, these results are intended to improve our understanding regarding the biomechanical aspects of the problem, which are thought to have a major contribution to the effective execution of DC. Accordingly, during the design of craniectomy, these results could be used as guidance which is associated with this biomechanical perspective. However, due to the presence of other medical circumstances which may govern the design, these observations should not be considered as ultimate optimums, always providing the best solution. In fact, these findings are the first results of the biomechanical optimization of DC (as far as the authors know), which may be continuously improved in the future. For instance, there are some aspects where the current FE modelling approach could be further improved in the future once hardware resources and new techniques become readily available even for this highly irregular and complex geometry. Some of these aspects could be the modelling of fluid-structure interaction between the tissue and the CSF and applying a time-dependent constitutive model. Contacts between the tissue and the skull could also be implemented as per our original modelling strategy, once remeshing techniques became more robust and the available hardware resources enable to run these large number of simulations series within a reasonable time. It is also mentioned, that although the current modelling strategy is advanced in the sense that the validation procedure included the consideration of displacements and ICPs too, strains were still not validated. It may be assumed that the obtained strains are reasonable if the simulated displacement field (both qualitatively and quantitatively) gives good agreement with the real-life observation. However, in the future, further measures should be performed to validate the strain field as well. This could be possible by the reconstruction of multiple real-life DC cases by nonlinear medical image registration [24]. Furthermore, currently, our results are limited due to the lack of knowledge regarding $T_{\text {DamE }}$ and $T_{\text {DamG }}$ thresholds of the brain tissue. Due to the above-mentioned parametric study, approximate results could be still obtained, but the need for further experimental research works, which aim to determine these thresholds corresponding to the circumstances of DC, must be highly emphasized. It is also noted that the current results come from the applied goals and penalty functions, which may be further improved and modified in the future based on the results of new medical studies. However, if later works reveal that the ratio between the three objective functions should be modified, then this can be done by adjusting the $w_{\text {ICP }}, w_{\text {DamE }}, w_{\text {DamG }}$ factors, which makes it possible to use our current results later on. As opposed to previous studies of tumour resection [19], prescribed displacements of the tissue could not be applied, because it would have been biased the observed bulging displacement and the corresponding pressure reduction. Unfortunately, this cause that the observed 
results are sensitive to the applied material model, which should be considered in the future to achieve further improvement in the direction of patientspecific optimization. Such an effort would be especially important due to the extremely large variability of the brain tissue properties [9]. It is mentioned that the current results were determined for a geometry where the area of the smoothed outer brain surface equalled to $600 \mathrm{~cm}^{2}$. For other geometries, the scaling of these results may be needed based on the ratio of the brain surfaces. Our current investigations could be extended to other types of skull openings and injury types in the future, with special attention to the fronto-temporoparietal opening and to cases where the intracranial hypertension is caused by subdural hematoma. In most cases, each patient-specific model offers only one validation possibility [12], [15] thus a large emphasis should be taken in the future on further validation possibilities using FE models and maybe other techniques as well. Nevertheless, it is believed that our currently presented results could provide the necessary basis for a continuous validation and modification procedure, which may lead to so robust results that can be implemented into the clinical practice in the future serving the ultimate goal of personalized medicine.

\section{Conclusions}

A comprehensive analysis of the biomechanical behaviour of the brain tissue during DC has been provided based on a large number of FE simulations. These results pointed out that the postoperative pressure has an approximately linear dependence on the skull opening area and the preoperative pressure. The currently detailed optimizations strategy enabled us to determine the optimal skull opening for several input parameters. These results indicated that the unilateral skull opening is more advantageous than the currently analysed bifrontal openings. The largescale parametric study pointed out that the optimal unilateral skull opening area usually falls within the $130-180 \mathrm{~cm}^{2}$ interval. Although, current results may be limited by the lack of knowledge of exact tissue resistance thresholds, these observations can provide guidance during the design of DC and the basis for a long-term validation procedure which one day may lead to the implementation of such an optimization into the clinical practice and definition of individualized therapeutic plans.

\section{Acknowledgement}

This work was supported by the Higher Education Excellence Program of the Ministry of Human Capacities in the frame of Biotechnology research area of Budapest University of Technology and Economics (BME FIKP-BIO).

\section{References}

[1] Amunts K., Lepage C., Borgeat L., Mohlberg H., DiCKSCHEID T., ROUSSEAU M.-É., BigBrain: An UltrahighResolution 3D Human Brain Model, Science, 2013, 340 (6139), 1472-1475.

[2] ANSYS $^{\circledR}$ Academic Research, Release 18.0, ANSYS Workbench User's Guide, ANSYS, Inc.

[3] CoOper D.J., Rosenfeld J.V., Murray L., Arabi Y.M., DAVIES A.R., D'Urso P. et al., Decompressive craniectomy in diffuse traumatic brain injury, New England Journal of Medicine, 2011, 364 (16), 1493-1502.

[4] ElKIN B., MORRISON B., Region-specific tolerance criteria for the living brain, Stapp Car Crash J., 2007, 51, 127-138.

[5] Faul M., Xu L., Wald M.M., Coronado V.G., Traumatic Brain Injury in the United States: Emergency Department Visits, Hospitalizations and Deaths 2002-2006, Centers for Disease Control and Prevention, National Center for Injury Prevention and Control, Atlanta,GA, 2010.

[6] Fletcher T.L., Kolias A.G., Hutchinson P.J.A., Sutcliffe M.P.F., Development of a Finite Element Model of Decompressive Craniectomy, PLoS One, 2014, 9 (7), e102131.

[7] Fletcher T.L., Wirthl B., Kolias A.G., AdAms H., HutChinson P.J.A., SuTClifFe M.P.F., Modelling of Brain Deformation After Decompressive Craniectomy, Annals of Biomedical Engineering, 2016, 44(12), 3495-3509.

[8] Fowler S.S., LeOnetti J.P., Banich J.C., LeE J.M., WURSTER R., Young M.R.I., Duration of neuronal stretch correlates with functional loss, Otolaryngol. Head Neck Surg., 2001, 124, 641-644.

[9] Franceschini G., Bigoni D., Regitnig P., Holzapfel G.A., Brain tissue deforms similarly to filled elastomers and follows consolidation theory, Journal of the Mechanics and Physics of Solids, 2006, 54, 2592-2620.

[10] GAO C.P., ANG B.T., Biomechanical modelling of decompressive craniectomy in traumatic brain injury, [in:] G.T. Manley, C. Hemphill, S. Stiver (Eds.), Acta Neurochirurgica Supplementum, Springer, Vienna, 2008, 102, 279-282.

[11] Hazay M., Bakos B., Tóth J.P., BüKi A., BojtáR I., Optimization of Decompressive Craniectomy based on Finite Element Simulations, [in:] D. Kytyr, Z. Major, T. Doktor (Eds.), 16th Youth Symposium on Experimental Solid Mechanics, Acta Polytechnica CTU Proceedings, 2018, May 17-19, Traunkirchen, Austria, 2018, 18, 6-9.

[12] Hazay M., VArga A., NAgy E., Tóth P.J., BüKi A., BoJTÁR I., Finite element reconstruction of decompressive craniectomy, Biomechanica Hungarica, 2018, 9 (2), 51-60.

[13] Horgan T.J., Gilchrist M.D., The creation of three-dimensional finite element models for simulating head impact biomechanics, I. J. Crash, 2003, 8 (4), 353-366.

[14] Hutchinson P.J., Kolias A.G., Timofeev I.S., Corten E.A., Czosnyka M., Тімотнy J. et al., Trial of Decompressive Craniectomy for Traumatic Intracranial Hypertension, New England Journal of Medicine, 2016, 375 (12), 1119-1130. 
[15] Li X., vOn Holst H., Finite Element Modeling of Decompressive Craniectomy $(D C)$ and its Clinical Validation, Advances in Biomedical Science and Engineering, 2015, 2 (1), 1-9.

[16] MaAs A.I., Roozenbeek B., Manley G.T., Clinical trials in traumatic brain injury: past experience and current developments, Neurotherapeutics, 2010, 7, 115-126.

[17] Marmarou A., Pathophysiology of traumatic brain edema: current concepts, Acta Neurochir. Suppl., 2003, 86, 7-10.

[18] Messac A., Optimization in Practice with MATLAB ${ }^{\circledR}$ for Engineering Students and Professionals, Cambridge University Press, New York (NY), 2015, 159-161.

[19] Miller K., Wittek A., TAVNer A., Joldes G., Biomechanical Modelling of the Brain for Neurosurgical Simulation and Neuroimage Registration, [in:] K. Miller (Ed.), Biomechanics of the Brain, Springer, 2019, 135-164.

[20] PiePer S., Halle M., Kikinis R., 3D Slicer, Proceedings of the 2nd IEEE International Symposium on Biomedical Imaging: Nano to Macro, 2004, Apr. 15-18, Arlington, VA, USA, 2004, 1, 632-635.
[21] Sahuquillo J., Decompressive craniectomy for the treatment of refractory high intracranial pressure in traumatic brain injury, Cochrane Database Syst. Rev., 2006, 1, CD003983.

[22] The MathWorks, MATLAB ${ }^{\circledR}$ Programming Fundamentals 2016b, The MathWorks, Natick (MA), 2016.

[23] Thurman D.J., Alverson C., Dunn K.A., Guerrero J., SNIEZEK J.E., Traumatic brain injury in the United States: A public health perspective, J. of Head Trauma Rehabil., 1999, 14 (6), 602-615.

[24] von Holst H., Li X., KLEIVEN S., Increased strain levels and water content in brain tissue after decompressive craniotomy, Acta Neurochir., 2012, 154 (9), 1583-1593.

[25] Wang F., Han Y., Wang B., Peng Q., Huang X., Miller K., WitTeK A., Prediction of brain deformations and risk of traumatic brain injury due to closed-head impact: quantitative analysis of the effects of boundary conditions and brain tissue constitutive model, Biomechanics and Modeling in Mechanobiology, 2018, 17 (4), 1165-1185. 